\title{
Future expectations in clinical depression: biased or realistic?
}

\author{
Ulrike Zetsche $^{1}$, Paul-Christian Bürkner ${ }^{2}$, Babette Renneberg ${ }^{1}$
}

1 Freie Universität Berlin, Clinical Psychology and Psychotherapy, Berlin, Germany

2 University of Münster, Department of Statistics, Faculty of Psychology, Münster, Germany

* Corresponding author. Ulrike Zetsche, Department of Clinical Psychology and Psychotherapy, Freie Universität Berlin, Habelschwerdter Allee 45.14195 Berlin, Germany, Phone: +49-30-838 55925, Fax: +49-30-838-51233; E-mail address: u.zetsche@ @u-berlin.de 


\begin{abstract}
Negative expectations have been highlighted as a key mechanism in the maintenance and recurrence of depression. Nonetheless, no study has investigated depressed individuals' expectations about their future mood so far. This is surprising given that experiences of sustained negative mood and lack of positive mood are the hallmark symptoms of depression. The present study thus assessed depressed individuals' expectations about their future mood and examined whether these expectations are accurate or negatively biased.

The study used experience sampling methodology (ESM) to contrast participants' expectations with their actually experienced sad and happy mood within a four-day period. At the end of this period, participants recalled their past mood. All variables were assessed in 30 clinically depressed individuals and 37 matched healthy controls, as well as in 56 undergraduate students with a wide range in depressive symptoms.

Results revealed that clinically depressed individuals held negatively biased expectations about their future mood: they expected more sad and less happy mood than they actually experienced. In contrast, healthy individuals showed realistic expectations about their future mood. Depressed individuals also demonstrated a negative mood recall bias. Finally, individuals' expectations and memories were more closely linked to depression status than their actually experienced mood.
\end{abstract}

Although negative mood is the hallmark symptom of depression, the expectations and memories of negative mood may be even more central for the understanding of depression.

Keywords: Expectations, Affective Forecasting, Depression, Experience Sampling Methodology, Memory Bias 


\section{Future expectations in clinical depression}

Negative expectations play a key role in the maintenance of mental disorders such as major depression. The central role of negative expectations for psychopathology has recently been reiterated by Rief and colleagues (Rief et al., 2015) and has long been proposed by cognitive theories of depression. For example, Beck and colleagues (e.g., Beck \& Haigh, 2014; Beck, Rush, Shaw, \& Emery, 1979) have postulated that depressed individuals are characterized by a negatively biased view of themselves and the world, as well as by negative expectations about their future. Such negative future expectations are harmful if they guide depressed individuals' behavior in a way that maintains depressed states. For example, if depressed individuals expect that they will feel down and despondent on the weekend, they may cancel activities with friends, thereby reducing opportunities for uplifting experiences. In addition, negative expectations about one's future mood may make it harder to bear negative mood states: if a person is feeling down and expects that this mood state will persist, it will be hard to accept it. Dealing with negative mood states will be easier if one expects that they will change in a while. Negative expectations about one's future mood states may thus be an important risk factor for the maintenance and recurrence of depression. Surprisingly, however, there have been no studies examining clinically depressed individuals' expectations about their future mood.

An important question in this context is whether depressed individuals' future expectations are accurate. Alloy and Abramson (1988) have postulated that depressed individuals exhibit a realistic view of the future (depressive realism). Accordingly, depressed individuals' expectations about their future mood may be negative but realistic. In contrast, Beck's cognitive theory of depression postulates that depressed individuals show negatively biased expectations about their future. According to this theory, depressed individuals would expect their negative mood to be worse than it actually will be. A differentiation of these two theories will have important clinical implications: if depressed individuals primarily exhibit a negatively biased 
view of their future mood states, it will be important to emphasize cognitive techniques correcting this bias (Rief et al., 2015).

Surprisingly, past research has paid little attention to depressed individuals' expectations about their future mood. There have been two studies assessing expectations about future mood (i.e., mood forecasting) in non-clinical undergraduate samples: Wenze and colleagues (Wenze, Gunthert, \& German, 2012) asked an unselected sample of undergraduate students to indicate how they expected to feel during the upcoming week using four positive and seven negative mood items. Subsequently, students rated their actual mood four times a day during the respective week. At the end of the week, students' memories of their past mood were assessed. Results showed that students' level of depressive symptoms was associated with higher forecasted, actual, and recalled negative mood and with lower forecasted, actual, and recalled positive mood. To examine whether students' mood forecasts and memories were accurate, the authors computed difference scores between forecasted and actual mood levels, and between recalled and actual mood levels. Students' level of depressive symptoms was associated with higher forecasting inaccuracy for positive mood, and higher recall inaccuracy for negative and positive mood. Interestingly, participants' level of depressive symptoms were only associated with forecasting inaccuracy for sad mood, but not for the sum score across all negative mood items.

In addition, Hoerger and colleagues (Hoerger, Quirk, Chapman, \& Duberstein, 2012) assessed mood forecasts for Valentine's Day and the following two days in a large undergraduate sample and compared those forecasts with the actually experienced mood on these days. Similar to the results by Wenze and colleagues (2012), the authors found that students' depressive symptoms were associated with higher predicted and actual negative mood, and with lower predicted and actual positive mood. Furthermore, depressive symptom levels were associated with forecasting inaccuracy for positive and negative mood as assessed by differences scores between forecasted and actual mood levels. 
Finally, a recent study by Thompson and colleagues (2017) assessed mood forecasting and actually experienced mood in remitted depressed individuals. They found that remitted depressed individuals expected less positive and more negative mood than never-depressed controls, but also experienced less positive and more negative mood than never-depressed controls. To examine whether depressed individuals expectations were less accurate than those of healthy controls, the authors tested the effect of diagnostic group on correlations between expected and actual mood levels. Results showed that the correlations between expected and actual mood levels did not differ between diagnostic groups.

The present research project is the first to examine mood forecasting in currently depressed individuals and matched healthy controls. Similar to the study by Wenze and colleagues (2012), we used experience sampling methodology to contrast participants' expectations with their actual mood levels during a subsequent four-day period. In addition, participants recalled their past mood levels at the end of the four-day period. Given that depression-related forecasting errors in the study by Wenze et al. (2012) were specific to sad mood (versus general negative mood), we assessed sad and happy mood levels separately. Following the proposition that negative expectations are at the core of mental disorders, the present research project finally examined whether depressive symptom levels are more strongly associated with distorted expectations and memories, or with actual experiences of disturbed mood. The project comprises of two studies using the same design: a pilot study assessing a student sample with a wide range in depressive symptoms (Student Study), and the main study assessing individuals with a current major depressive episode as well as matched healthy controls (Clinical Study).

We expected that diagnostic status or depressive symptoms, respectively, would be associated a) with higher forecasted sad mood and lower forecasted happy mood, b) with higher actually experienced sad mood and lower actual happy mood, and c) with negatively biased forecasts of sad and happy mood levels (Hoerger et al., 2012; Wenze et al., 2012). Based on extensive evidence for a negative memory bias in depression (for a review, see Matt, Vázquez, 
\& Campbell, 1992), we further expected that diagnostic status or depressive symptoms, respectively, would be associated with a negative memory bias for sad and happy mood levels.

\section{Methods}

\section{Participants}

Student study. $\mathrm{N}=56$ students took part in the study. They were on average 25.6 (SD = 4.56) years old and $82.1 \%$ were female. The sample's average score on the German version of the Center for Epidemiological Studies - Depression Scale (German version: Hautzinger \& Bailer, 1993; CES-D; Radloff, 1977) was $18.8(S D=11.3)$ ranging from 4 to 47 . One third of participants $(\mathrm{N}=18,32.1 \%)$ had a CES-D score above 22 , which is the cut-off for clinical depression. $\mathrm{N}=3$ participants did not return for the second lab session resulting in a sample size of $\mathrm{N}=53$ for all analyses regarding mood recall.

Given that the student study was part of a joint project examining cognitive processes in depressed and socially anxious students, participants interested in the study were screened for low and high degrees of social anxiety using the German version of the Social Phobia Inventory (SPIN; Connor et al., 2000; German version: Sosic, Gieler, \& Stangier, 2008). To compensate for a possible selection bias, we included SPIN scores as a control variable in all analyses of this sample. The sample showed a mean SPIN score of $18.5(\mathrm{SD}=14.7)$.

Clinical study. The clinical study comprised of participants meeting diagnostic criteria for a current major depressive episode and healthy individuals with no past or present mental disorder. The presence or absence of mental disorders was established by the structured clinical interview for DSM IV (German Version: Wittchen, Wunderlich, Gruschwitz, \& Zaudig, 1997). All participants had to be between 18 and 65 years old. Depressed participants were excluded if they met criteria for any psychotic, bipolar, or substance dependence disorder. 
Thirty-four individuals with a present major depressive episode and 41 matched healthy individuals took part in the study. $\mathrm{N}=3$ individuals ( 2 depressed, 1 control) dropped out after the clinical interview. $\mathrm{N}=2$ participants did not have any ambulatory assessment data due to technical problems and another $\mathrm{N}=3$ participants were excluded from data analyses because they only completed 2, 4, and 7 out of 20 ambulatory assessment prompts, respectively. All other participants completed at least $50 \%$ of prompts. Thus, the final sample comprised of 30 individuals with a major depressive episode and 37 healthy controls. Demographic and clinical information is depicted in Table 1. Groups did not differ in age or gender.

Table 1.

Descriptive statistics across the entire sample and by diagnostic group in the clinical study

$\begin{array}{lll}\text { Variables } & \text { Total sample } \quad \text { Depressed Group Control Group }\end{array}$

\begin{tabular}{|c|c|c|c|c|c|c|}
\hline & Number & $\%$ & Number & $\%$ & Number & $\%$ \\
\hline \multirow[t]{2}{*}{ Female Participants } & 55 & 82.10 & 25 & 83.30 & 30 & 81.10 \\
\hline & Mean & SD & Mean & SD & Mean & SD \\
\hline Age & 35.00 & 9.98 & 34.87 & 10.85 & 35.11 & 9.37 \\
\hline CES-D score & 19.60 & 15.01 & 34.47 & 8.50 & 7.54 & 4.59 \\
\hline Nr. of episodes & - & - & 1.70 & 0.47 & - & - \\
\hline
\end{tabular}

Note: $S D$ = Standard deviation; CES-D = Center for Epidemiological Studies - Depression Scale

\section{Measures}

Depressive symptoms. In both studies, the German version of the Center for Epidemiological Studies - Depression Scale (German version: Hautzinger \& Bailer, 1993; CES-D; Radloff, 1977) was used to assess level of depressive symptoms within the past week. The German version of the CES-D comprises 20 items assessing emotional, motivational, 
cognitive, and somatic symptoms of depression. The total score may vary between $0-60$ and the cut-off score for clinical depression is 22 (Hautzinger, Bailer, Hofmeister, \& Keller, 2012). The German version of the CES-D shows good psychometric properties (Hautzinger et al., 2012).

Mood expectation. Prior to the ambulatory assessment period, participants in both studies were presented with the following six emotion adjectives and asked to indicate on a scale from 1 (not at all) to 5 (very much) how much they expected to feel this way on average during the following four days: sad, downhearted, nervous, insecure, cheerful, and carefree. Adjectives were taken from the German version of the PANAS-X (Grühn, Kotter-Grühn, \& Röcke, 2010; Watson \& Clark, 1999). Mood forecasting scores were computed for sad mood (mean rating for sad and downhearted, Cronbach's $\alpha=.89$ in the student study; Cronbach's $\alpha$ $=.92$ in the clinical study); happy mood (cheerful, carefree, Cronbach's $\alpha=.67$ in the student study; Cronbach's $\alpha=.77$ in the clinical study), and fearful mood (nervous, insecure, Cronbach's $\alpha=.65$ in the student study; Cronbach's $\alpha=.86$ in the clinical study). Note, that all analyses regarding fearful mood levels are presented in the online supplements as we did not have specific hypotheses regarding fearful mood.

Actually experienced mood. In both studies, actual daily mood was assessed using ambulatory assessment over a period of four days (Tue - Fri). For this purpose, participants received a Motorola G5 with an ambulatory assessment application. Prompts occurred five times a day between 10 am and 8 pm following a semi-random order (i.e., participants received a total of 20 prompts). At each prompt, participants were presented with the same six emotion adjectives and asked how much they were currently feeling this way on a scale from 1 (not at all) to 7 (very much). This scale was later rescaled to range from 1-5 to make it comparable to the mood forecasting and recall values. Given that the scales' anchors were identical, this is a linear transformation from 1-7 to 1-5. Participants were allowed to delay responses by max. 15 minutes. At the end of each day, an additional prompt asked 
participants to indicate which of 16 events had occurred that day (data not reported here). Participants were informed about an incentive of $5 €$ or equivalent course credit if they completed at least $90 \%$ of the prompts. The average response rate to prompts across all participants was $87.8 \%$ in the student study, and $88.5 \%$ in the clinical study. Neither CES-D nor SPIN scores were correlated in a relevant manner with the number of completed prompts in the student study, all $|r|<.17$. Similarly, the response rate to prompts did not differ between groups in the clinical study (MDD group: $88.4 \%$, control group: $88.7 \%$ ).

Mood recall. After the ambulatory assessment period, participants in both studies were presented with the six emotion adjectives again and asked to indicate how much they had been feeling this way on average during the past four days. Mood recall scores were computed for sad mood (mean rating for sad and downhearted, Cronbach's $\alpha=.81$ in the student study; Cronbach's $\alpha=.92$ in the clinical study), happy mood (cheerful, carefree, Cronbach's $\alpha=.75$ in the student study; Cronbach's $\alpha=.74$ in the clinical study), and fearful mood (nervous, insecure, Cronbach's $\alpha=.84$ in the student study; Cronbach's $\alpha=.92$ in the clinical study).

\section{Procedure}

Both studies consisted of two lab sessions and a four-day ambulatory assessment period in-between lab sessions. Participants in the clinical study also took part in a clinical interview prior to the first lab session. The first lab session was always on Mondays, so that the ambulatory assessment took place on weekdays (Tue to Fri). In the first lab session, participants provided written informed consent and completed the mood prediction and a set of questionnaires including the CES-D. At the end of the four-day period, students returned to the lab to rate their recalled mood during the ambulatory assessment period. They also completed other tasks not part of this study. Participants in the clinical study rated their recalled mood in the past four days via an online questionnaire. All participants were 
compensated for their time. Both studies were approved by the Ethics Committee of the Freie University Berlin.

\section{Statistical Analyses}

We estimated Bayesian regression models to examine our hypotheses. For this purpose, we used the R (R-Core-Team, 2018) package brms (Bürkner, 2017a, 2017b), which is based on Stan (Carpenter et al., 2017).

For all analyses predicting ambulatory assessment variables, i.e., variables with several data points per person, we estimated Bayesian linear multilevel models with varying intercepts over persons. For all analyses predicting person-level variables, i.e., variables with only one data point per person, we estimated Bayesian linear regression models. For all analyses including the group factor (in the clinical sample), we modelled the variance to vary across groups. As priors we always used the default priors of brms, which are chosen to be non- or weakly informative, thus having only negligible influence on the obtained results (Bürkner, 2017a, 2017b). Predictor variables in all analyses were standardized. Please see the results section for the specific dependent and independent variables in each analysis. Effects were considered clearly different ${ }^{1}$ from zero if the estimate's $95 \%$ credible interval (i.e., Bayesian confidence interval) did not include zero. For directed hypotheses, we also estimated the posterior probability that the respective effect is in the expected direction.

To examine whether or not participants' expectations or memories of their actually experienced mood were accurate, we computed individual differences scores between forecasted and experienced mood levels, and between recalled and experienced mood levels, respectively (Wenze, et al., 2012). For this purpose, we computed difference scores by subtracting the actual mood levels from the forecasted or recalled mood levels, respectively. These difference scores indicate the degree to which participants overestimate (positive error scores) or underestimate (negative error scores) their future or past mood levels relative to their actually experienced mood levels. 
RUNNING HEAD: FUTURE EXPECTATIONS DEPRESSION

All data, $\mathrm{R}$ code, and results are available at

https://osf.io/tk3fm/?view_only=2e39ac0b172c44a68d6b3512aaf5b33e. 


\section{Results}

\section{Descriptive Statistics}

Table 2 displays average expected, actually experienced, and recalled mood levels in the student and clinical study. It also displays average expectation and recall errors in the two studies.

Table 2

Means and standard deviations for all relevant variables in the two studies

\begin{tabular}{|c|c|c|c|c|c|c|c|c|}
\hline Variables & \multicolumn{2}{|c|}{ Student study } & \multicolumn{2}{|c|}{$\begin{array}{l}\text { Total sample } \\
\text { clinical study }\end{array}$} & \multicolumn{2}{|c|}{$\begin{array}{l}\text { Depressed } \\
\text { Group }\end{array}$} & \multicolumn{2}{|c|}{$\begin{array}{l}\text { Control } \\
\text { Group }\end{array}$} \\
\hline Expectations $(1-5)^{1}$ & Mean & SD & Mean & SD & Mean & SD & Mean & SD \\
\hline Sad mood & 2.12 & 1.09 & 2.22 & 1.18 & 3.27 & 0.94 & 1.38 & 0.48 \\
\hline Happy mood & 2.88 & 0.87 & 2.82 & 1.10 & 1.92 & 0.82 & 3.55 & 0.67 \\
\hline \multicolumn{9}{|l|}{ Actual mood $(1-5)^{1,2}$} \\
\hline Sad mood & 1.87 & 0.56 & 1.88 & 0.78 & 2.60 & 0.53 & 1.30 & 0.32 \\
\hline Happy mood & 2.99 & 0.61 & 2.85 & 0.77 & 2.28 & 0.48 & 3.32 & 0.63 \\
\hline \multicolumn{9}{|l|}{ Recall $(1-5)^{1}$} \\
\hline Sad mood & 2.28 & 1.02 & 2.37 & 1.18 & 3.47 & 0.61 & 1.47 & 0.64 \\
\hline Happy mood & 2.99 & 0.96 & 2.84 & 1.01 & 1.97 & 0.60 & 3.55 & 0.65 \\
\hline \multicolumn{9}{|l|}{ Expectation error ${ }^{2}$} \\
\hline Sad mood & 0.26 & 0.84 & 0.35 & 0.76 & 0.64 & 0.94 & 0.11 & 0.46 \\
\hline Happy mood & -0.10 & 0.65 & -0.03 & 0.80 & -0.33 & 0.74 & 0.21 & 0.76 \\
\hline \multicolumn{9}{|l|}{ Recall error $^{2}$} \\
\hline Sad mood & 0.39 & 0.70 & 0.49 & 0.60 & 0.83 & 0.60 & 0.21 & 0.44 \\
\hline Happy mood & -0.01 & 0.60 & -0.01 & 0.52 & -0.27 & 0.52 & 0.20 & 0.42 \\
\hline
\end{tabular}

Note: $S D=$ Standard deviation; $1=$ scale range from $1-5 ; 2=$ group means and SDs of individuals intercepts. Intercepts are estimated from Bayesian linear multilevel null-models with varying intercepts over persons. 


\section{Expectations about Future Mood}

Student study. We estimated Bayesian linear regression models with expected mood levels as dependent variable and students' depressive and social anxiety symptoms as independent variables. Results showed that students with higher depressive symptoms expected clearly higher levels of sad mood, $b=0.95(95 \% C I=[0.73,1.17])$, and lower levels of happy mood, $b=-0.54(95 \% C I=[-0.77,-0.31])$, (see Table S1a in the online supplements for details).

Clinical study. To examine the effect of diagnostic status on expected mood levels, we estimated Bayesian linear regression models with expected mood scores as dependent variable and group as independent variable. Results showed that depressed individuals expected clearly higher levels of sad mood, $b=1.89(95 \% C I=[1.51,2.27])$, and lower levels of happy mood, $b=-1.64(95 \% C I=[-2.02,-1.26])$, than their healthy counterparts (see Table S1b in the online supplements for details).

\section{Actually Experienced Mood}

Student study. To examine the association between students' daily mood levels and their level of depressive symptoms, we estimated Bayesian linear multilevel models with daily mood levels as dependent variable and levels of depressive and social anxiety symptoms as independent variables. Students' level of depressive symptoms showed a clear positive association with daily sad mood levels, $b$ CESD $=0.38(95 \% C I=[0.21,0.54])$, and a clear negative association with daily happy mood levels, $b_{\mathrm{CESD}}=-0.36(95 \% C I=[-0.55,-0.18])$, (see Table S2a in the online supplements for details). Social anxiety symptoms were not associated with daily mood levels.

Clinical study. We estimated Bayesian linear multilevel models to predict individuals' daily mood levels by their diagnostic status. Clinically depressed individuals showed clearly higher daily sad mood levels, $b$ MDD $=1.34(95 \% C I=[1.13,1.56])$, and clearly lower daily 
happy mood levels than healthy controls, $b_{\mathrm{MDD}}=-1.09(95 \% C I=[-1.38,-0.79])$, (see Table $\mathrm{S} 1 \mathrm{~b}$ in the online supplements for details).

\section{Recalled Mood}

Student study. Bayesian linear regression models with recalled mood levels as dependent variable and students' depressive and social anxiety symptoms as independent variables showed that students with higher depressive symptoms recalled clearly higher levels of sad mood, $b=0.78(95 \% C I=[0.56,1.00])$, and lower levels of happy mood, $b=-0.50$ $(95 \% C I=[-0.77,-0.23])$, (see Table S1a in the online supplements for details).

Clinical study. Bayesian linear regression models with recalled mood scores as dependent variable and group as independent variable showed that depressed individuals recalled clearly higher levels of sad mood, $b=2.00(95 \% C I=[1.69,2.31])$, and lower levels of happy mood than their healthy counterparts, $b=-1.59$ (95\% $C I=[-1.90,-1.28])$, (see Table $\mathrm{S} 1 \mathrm{~b}$ in the online supplements for details).

\section{Expectation and Recall Errors}

Next, we wanted to examine whether or not depressed individuals' negative appraisal of their future and past mood is accurate. For this purpose, we computed difference scores between their expected and actually experienced mood levels, and between their recalled and experienced mood levels (see Methods section for details). These difference scores indicate the degree to which participants overestimate (positive error scores) or underestimate (negative error scores) their future or past mood levels relative to their actually experienced mood levels.

Student study. Bayesian linear multilevel models with the respective error score as dependent variable and CES-D and SPIN scores as predictors demonstrated that students' level of depressive symptoms was positively associated with an overestimation of future and past sad mood. Depressive symptom levels were only weakly associated with errors in the 
expectation or recall of happy mood. Detailed results are displayed in Table 3a and illustrated in Figure 1a.

Table 3a

Prediction of students' expectation and recall errors by their levels of depressive and social anxiety symptoms

\begin{tabular}{lllll} 
Variables & Estimate & SE & CI95lower/upper & Post. Prob. \\
\hline Expectation Errors & & & & \\
$d V$ : Sad mood & & & & \\
Intercept & 0.26 & 0.10 & $0.06,0.46$ & 1.00 \\
CES-D & 0.57 & 0.13 & $0.31,0.83$ & \\
SPIN & -0.14 & 0.13 & $-0.40,0.11$ & \\
\hline$d V:$ Happy mood & & & & 0.93 \\
Intercept & -0.10 & 0.10 & $-0.29,0.09$ & \\
CES-D & -0.17 & 0.12 & $-0.41,0.07$ & \\
SPIN & -0.02 & 0.12 & $-0.25,0.22$ & \\
\hline Recall Errors & & & & \\
\hline
\end{tabular}

\section{Recall Errors}

\begin{tabular}{lcclc}
\hline$d V:$ Sad mood & & & & \\
Intercept & 0.38 & 0.09 & $0.20,0.55$ & 1.00 \\
CES-D & 0.41 & 0.11 & $0.18,0.63$ & \\
SPIN & 0.00 & 0.11 & $-0.22,0.22$ & \\
\hline$d V:$ Happy mood & & & & 0.89 \\
Intercept & 0.00 & 0.09 & $-0.19,0.19$ & \\
CES-D & -0.14 & 0.12 & $-0.37,0.09$ & \\
SPIN & -0.05 & 0.11 & $-0.27,0.17$ &
\end{tabular}

Note: Prost. Prob. $=$ posterior probability that the estimate is in the expected direction; $C 195$

$=95 \%$ credible interval; $C E S-D=$ Center for epidemiological studies - depression scale;

SPIN = Social Phobia Inventory 

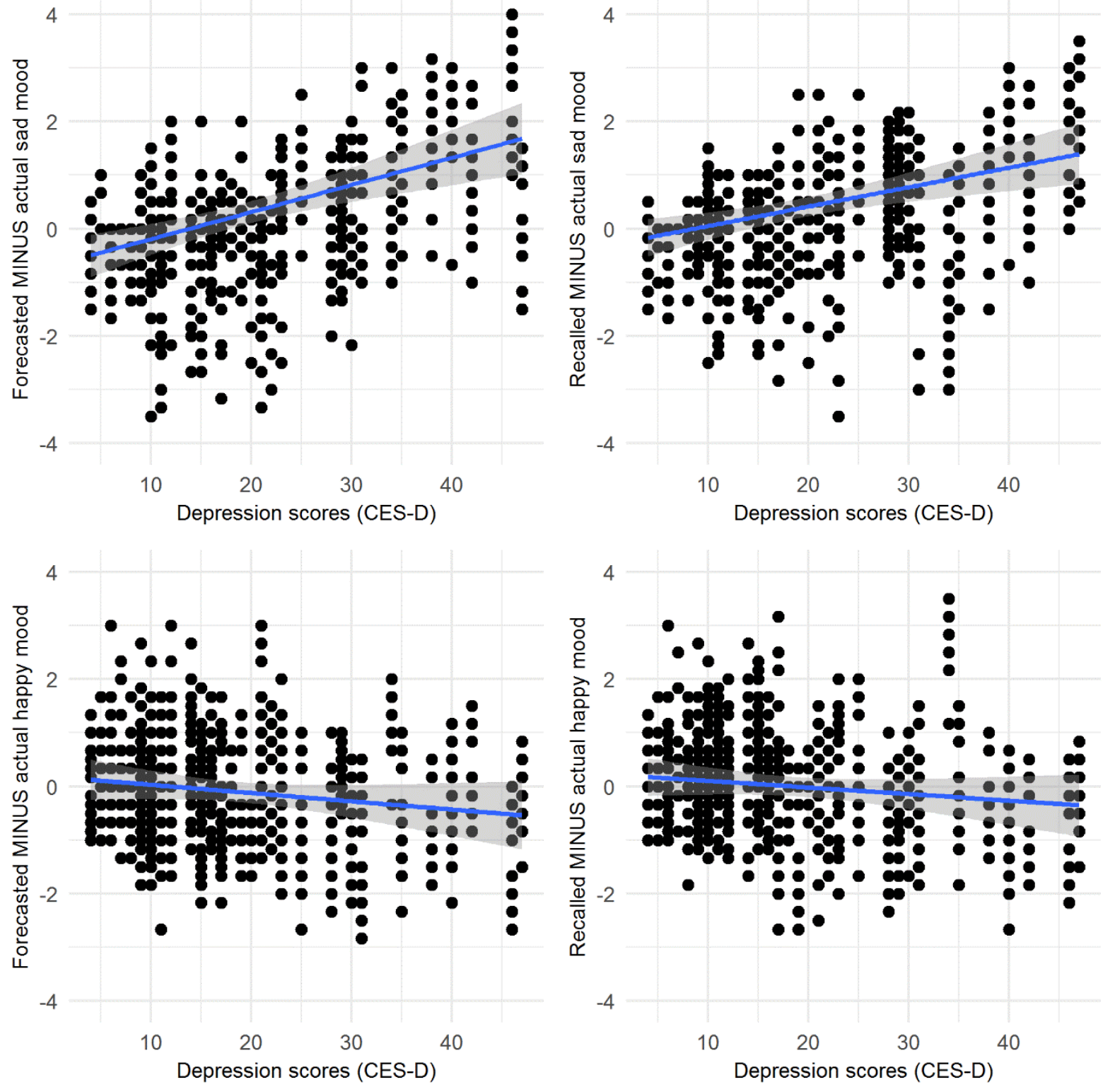

Figure 1a. Association between depressive symptoms and expectation and recall errors, controlling for SPIN scores in the student study. Dots represent data points, blue line represents estimated regression line, and gray area represents $95 \%$ credible interval of the regression line.

Clinical study. We estimated Bayesian linear multilevel models to examine the effect of diagnostic group on the respective error scores. Results are depicted in Figure $1 \mathrm{~b}$ and detailed in Table 3b. Depressed individuals overestimated their future sad mood and underestimated their future happy mood to a greater extent than healthy individuals. Likewise, depressed 
individuals overestimated their past sad mood and underestimated their past happy mood to a greater extent than healthy individuals. To examine whether expectations are accurate or distorted, we further tested whether the mean error scores in the two groups clearly differ from zero (see Table 3b). On average, depressed individuals expected to feel considerably sadder and less happy than they actually did. Depressed individuals also recalled to have felt considerably worse than they actually did. In contrast, healthy individuals forecasted their sad and happy mood levels rather realistically. However, they recalled to have felt sadder but also happier than they actually did. 
RUNNING HEAD: FUTURE EXPECTATIONS DEPRESSION

Table $3 b$

Expectation and recall error scores in the depressed and control group, and group difference in these error scores

\section{$\begin{array}{llll}\text { Variables } & \text { Estimate } & \text { SE } & \text { CI95lower/upper Post. Prob. }\end{array}$}

\section{Expectation Errors}

\begin{tabular}{|c|c|c|c|c|}
\hline Control group & 0.10 & 0.12 & $-0.13,0.34$ & \\
\hline Depressed group & 0.65 & 0.14 & $0.38,0.92$ & \\
\hline Group difference & 0.54 & 0.18 & $0.19,0.90$ & 1.00 \\
\hline \multicolumn{5}{|l|}{ Happy mood } \\
\hline Control group & 0.22 & 0.13 & $-0.04,0.49$ & \\
\hline Depressed group & -0.34 & 0.14 & $-0.61,-0.05$ & \\
\hline Group difference & -0.56 & 0.19 & $-0.92,-0.17$ & 1.00 \\
\hline
\end{tabular}

\section{Recall Errors}

\begin{tabular}{|c|c|c|c|c|}
\hline Sad mood & & & & \\
\hline Control group & 0.20 & 0.09 & $0.02,0.37$ & \\
\hline Depressed group & 0.85 & 0.10 & $0.65,1.05$ & \\
\hline Group difference & 0.65 & 0.14 & $0.38,0.92$ & 1.00 \\
\hline Happy mood & & & & \\
\hline Control group & 0.22 & 0.09 & $0.05,0.39$ & \\
\hline Depressed group & -0.29 & 0.10 & $-0.48,-0.09$ & \\
\hline Group difference & -0.50 & 0.13 & $-0.765-0.26$ & 1.00 \\
\hline
\end{tabular}



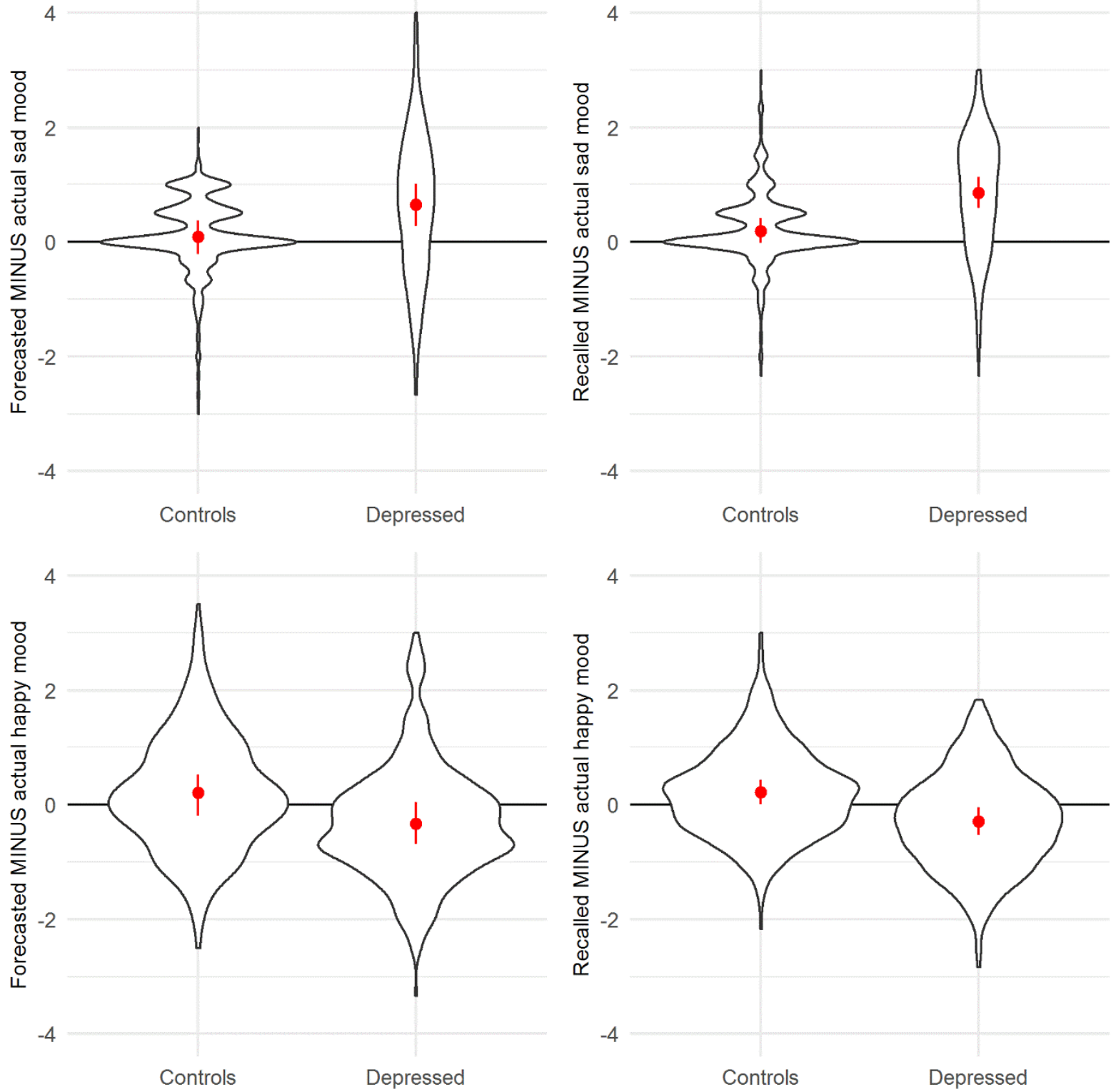

Figure $1 b$. Expectation and Recall errors in the depressed and control group. Violin plots represent actual data. Red dots and lines represent model estimates for the group means and their $95 \%$ credible intervals.

\section{Actual Experiences versus Expectations and Memories}

Finally, we wanted to examine whether depression is more strongly associated with actually experienced mood levels or with individuals' expectancies and memories of their mood. We thus estimated Bayesian linear regression models with depressive symptoms as 
dependent variable, and expected and recalled mood levels as well as the individual intercepts of the actually experienced mood levels as predictors. Due to the limited size of both samples, we could not include all mood scales into one analyses. For this purpose, we estimated one regression model with expected, experienced, and recalled sad mood levels as predictors, and another model with expected, experienced, and recalled happy mood levels as predictors. SPIN scores were entered as additional predictor in all models in the student sample.

In the student study, participants' level of depressive symptoms was associated with expected sad mood levels, $\beta=0.48(95 \% C I=[0.32,0.65])$, recalled sad mood levels, $\beta=$ $0.40(95 \% C I=[0.21,0.59])$, and SPIN scores, $\beta=0.20(95 \% C I=[0.06,0.34])$. Importantly, participants' level of depressive symptoms was not associated with higher levels of experienced sad mood when controlling for the other predictors, $\beta=-0.01(95 \% \mathrm{CI}=[-0.19$, 0.17]). In fact, the regression coefficients of expected and recalled mood levels were clearly larger than the regression coefficient of experienced sad mood: difference from expected mood coefficient $\beta=0.49(95 \% C I=[0.27,0.77])$; difference from recalled mood coefficient $\beta=0.41(95 \% C I=[0.15,0.72])$. In total, the model explained $81 \%$ of variance in depressive symptoms.

When entering happy mood variables as predictors, the level of expected happy mood was the only clear predictor of depressives symptoms, $\beta=-0.32(95 \% C I=[-0.59,-0.05])$. Recalled happy $\operatorname{mood} \beta=-0.09(95 \% \mathrm{CI}=[-0.39,0.21])$ or actual happy $\operatorname{mood} \beta=-0.23$ $(95 \% C I=[-0.53,0.06])$ were no clear predictors of depressive symptoms. Note, that the regression coefficient of expected happy mood levels was not clearly larger than that of experienced happy mood levels, difference $\beta=-0.09(95 \% C I=[-0.53,0.36])$. In total, the model explained $58 \%$ of variance in depressive symptoms.

In the clinical study, depressive symptom levels were predicted by higher expected sad mood, $\beta=0.35(95 \% C I=[0.19,0.51])$, higher recalled sad mood, $\beta=0.44(95 \% C I=[0.23$, $0.65])$, and higher experienced sad mood, $\beta=0.21(95 \% C I=[0.00,0.40])$. Although the 
regression coefficient of experienced sad mood was smaller than those of expected and recalled sad mood, this difference was not clear: difference from expected mood coefficient $\beta$ $=0.14(95 \% C I=[-0.14,0.43])$; difference from recalled mood coefficient $\beta=0.23(95 \% C I$ $=[-0.14,0.61])$. The model explained $84 \%$ of variance in depressive symptoms.

When entering happy mood variables as predictors, expected, $\beta=-0.43(95 \% C I=[-$ $0.62,-0.25])$, and recalled happy mood, $\beta=-0.57(95 \% C I=[-0.82,-0.32])$, predicted depressive symptoms to a clearly greater extent than experienced happy mood, $\beta=0.09$ (95\% $C I=[-0.16,0.33])$. In fact, the regression coefficients of expected and recalled happy mood levels were clearly larger than the regression coefficient of experienced happy mood: difference from expected mood coefficient $\beta=-0.35(95 \% C I=[-0.62,-0.07])$; difference from recalled mood coefficient $\beta=-0.48(95 \% C I=[-0.68,-0.29])$. In total, the model explained $72 \%$ of variance in depressive symptoms. 


\section{Discussion}

Negative expectations have been highlighted as a key factor involved in the maintenance of depression (Beck \& Haigh, 2014; Rief et al., 2015). Nonetheless, surprisingly little research has investigated negative expectations in depression so far. The present study was the first to assess depressed individuals' expectations about their future mood and to examine whether these expectations are negatively biased or in accordance with their actually experienced mood. Results revealed that currently depressed individuals as well as students with elevated levels of depression symptoms held negative expectations about their future mood: they both expected more sad mood and less happy mood than non-depressed individuals. Although depressed individuals in both samples actually did experience more sad and less happy mood than healthy individuals, their expectations were negatively distorted. Specifically, currently depressed individuals expected to feel considerably worse, i.e. sadder and less happy than they actually did. In contrast, healthy individuals forecasted their future sad and happy mood pretty accurately. In the student sample, higher levels of depressive symptoms were also associated with a greater overestimation of sad mood, but not with a greater underestimation of happy mood. In sum, these results provide evidence against the depressive realism hypothesis and rather suggest that depression goes along with negatively biased expectations about the future. This is in accordance with findings of two previous studies in non-clinical student samples showing that elevated levels of depressive symptoms are associated with negatively biased expectations about one's future mood (Hoerger et al., 2012; Wenze et al., 2012). Note, that in contrast to these previous studies, depressive symptoms in the present student sample were not associated with biased expectations about participants' future happy mood. This may suggest that depression-related biases in the expectation of happy mood are somewhat less robust than biases in the expectation of sad mood. In line with this assumption, Hoerger et al. (2012) reported a weaker association between depressive symptoms and forecasting errors for positive mood $(r=.19)$ than between 
depressive symptoms and forecasting errors for negative mood $(r=.36)$. It may be possible that the experience of increased sad mood is more prominent in depressed individuals' mental representation than the lack of positive mood. This may explain why their expectations about their future sad mood are more distorted than their expectations about their future positive mood.

It is important to note that Thompson et al. (2017) found that remitted depressed individuals did not differ from healthy individuals in their forecasting accuracy for positive and negative mood. The authors conclude that mood forecasting is not affected after the remission of depressive episodes. However, Thompson et al. (2017) used a different approach to analyze forecasting accuracy than the other studies. They examined the effect of diagnostic group on correlations between predicted and actual mood. It is not clear whether results derived by such an approach are comparable with those derived by difference scores.

In line with extensive evidence for a negative memory biases in depression (e.g., Matt et al., 1992), the present study found a marked mood recall error in depression. Specifically, currently depressed individuals recalled considerably more sad and less happy mood than they had actually experienced. In contrast, healthy individuals slightly overestimated both their past happy and sad mood. In the current student sample, elevated levels of depressive symptoms were associated with a recall error for sad mood, but not for happy mood. This mirrors the pattern of results regarding their expectation errors and supports the notion that depression-related representations of happy mood experiences are not as heavily distorted than those of sad mood experiences. Expectation and recall errors regarding happy mood only seem to be apparent in clinical depression. Interestingly, healthy individuals even showed the opposite pattern and overestimated their past happy mood. This is in accordance with evidence for positive recall biases in healthy individuals (for a review, see Walker, Skowronski, \& Thompson, 2003). 
When looking at the whole picture of the present results, it becomes evident that depressed individuals' representations of their affective experiences (i.e., expectations and memories) are considerably distorted. Although depressed individuals do experience more sad and less happy mood than healthy individuals, their expectations and memories of their mood are much worse than their actual experiences. This prompts the question whether depression is more closely related to actually experienced mood levels, or to the expectations and memories of their mood. To answer this question, we regressed expected, recalled, and actual mood levels on depression status, or depressive symptoms, respectively. Results showed that the weakest predictor in each analysis was the actually experienced mood level. These results suggest that, although sustained negative mood and a lack of positive mood are the hallmark symptoms of depression, the expectations and memories of these mood disturbances may be even more central for the understanding of depression.

It will be intriguing for future research to examine in a longitudinal design whether negatively distorted expectations and memories about one's mood may be a stronger predictor for the course of depression than the actual mood disturbances. It is possible that depressed individuals recollect a general self-concept rather than specific experiences when asked about their future or past mood. This would be in line with memory research showing that depressed individuals recall overgeneralized autobiographical memories when asked about specific events (Williams et al., 2007). If this is the case, depressed individuals' expectation about their future mood might be relatively resistant to actual changes and improvements in mood (Kube, Rief, \& Glombiewski, 2017), which may put them at increased risk for the recurrence of depressed mood states. In sum, the present results have important clinical implications and suggest that the modification of negatively distorted expectations and memories about depressed individuals' affective experiences should be a major target in psychotherapy. 


\section{References}

Alloy, L. B., \& Abramson, L. Y. (1988). Depressive realism: Four theoretical perspectives. York, NY, US: Guilford Press.

Beck, A. T., \& Haigh, E. A. (2014). Advances in Cognitive Theory and Therapy: The Generic Cognitive Model*. Annual review of clinical psychology, 10, 1-24.

Beck, A. T., Rush, A., Shaw, B., \& Emery, G. (1979). Cognitive therapy of depression. Guilford, New York.

Bürkner, P.-C. (2017a). Advanced Bayesian Multilevel Modeling with the R Package brms. The R Journal, 10(1), 395-411.

Bürkner, P.-C. (2017b). brms: An R Package for Bayesian Multilevel Models Using Stan. Journal of Statistical Software, 80(1), 1-28. doi:doi:10.18637/jss.v080.i01

Carpenter, B., Gelman, A., Hoffman, M. D., Lee, D., Goodrich, B., Betancourt, M., . . . Riddell, A. (2017). Stan: A probabilistic programming language. Journal of Statistical Software, 76(1).

Connor, K. M., Davidson, J. R., Churchill, L. E., Sherwood, A., Weisler, R. H., \& FOA, E. (2000). Psychometric properties of the Social Phobia Inventory (SPIN) New selfrating scale. The British Journal of Psychiatry, 176(4), 379-386.

Grühn, D., Kotter-Grühn, D., \& Röcke, C. (2010). Discrete affects across the adult lifespan: Evidence for multidimensionality and multidirectionality of affective experiences in young, middle-aged and older adults. Journal of Research in Personality, 44(4), 492500.

Hautzinger, M., \& Bailer, M. (1993). Allgemeine Depressionsskala (ADS). Deutsche Form der Center for Epidemiologic Studies Depression Scale (CES-D). Beltz Text. 
Hautzinger, M., Bailer, M., Hofmeister, D., \& Keller, F. (2012). ADS. Allgemeine Depressionsskala (2., überarbeitete und neu normierte Auflage). Manual. Göttingen: Hogrefe.

Hoerger, M., Quirk, S. W., Chapman, B. P., \& Duberstein, P. R. (2012). Affective forecasting and self-rated symptoms of depression, anxiety, and hypomania: Evidence for a dysphoric forecasting bias. Cognition \& Emotion, 26(6), 1098-1106.

Kube, T., Rief, W., \& Glombiewski, J. A. (2017). On the maintenance of expectations in major depression-investigating a neglected phenomenon. Frontiers in Psychology, 8, 9.

Matt, G. E., Vázquez, C., \& Campbell, W. K. (1992). Mood-congruent recall of affectively toned stimuli: A meta-analytic review. Clinical Psychology Review, 12(2), 227-255. doi:http://dx.doi.org/10.1016/0272-7358(92)90116-P

R-Core-Team. (2018). $R$ : A language and environment for statistical computing. $R$ Foundation for Statistical Computing. Vienna, Austria.

Radloff, L. S. (1977). The CES-D scale a self-report depression scale for research in the general population. Applied psychological measurement, 1(3), 385-401.

Rief, W., Glombiewski, J. A., Gollwitzer, M., Schubö, A., Schwarting, R., \& Thorwart, A. (2015). Expectancies as core features of mental disorders. Current opinion in psychiatry, 28(5), 378-385.

Sosic, Z., Gieler, U., \& Stangier, U. (2008). Screening for social phobia in medical in-and outpatients with the German version of the Social Phobia Inventory (SPIN). Journal of Anxiety Disorders, 22(5), 849-859.

Thompson, R. J., Spectre, A., Insel, P. S., Mennin, D., Gotlib, I. H., \& Gruber, J. (2017). Positive and negative affective forecasting in remitted individuals with bipolar I disorder, and major depressive disorder, and healthy controls. Cognitive Therapy and Research, 41(5), 673-685. 
Walker, W. R., Skowronski, J. J., \& Thompson, C. P. (2003). Life is pleasant--and memory helps to keep it that way! Review of General Psychology, 7(2), 203.

Watson, D., \& Clark, L. A. (1999). The PANAS-X: Manual for the positive and negative affect schedule-expanded form.

Wenze, S. J., Gunthert, K. C., \& German, R. E. (2012). Biases in affective forecasting and recall in individuals with depression and anxiety symptoms. Personality and Social Psychology Bulletin, 38(7), 895-906.

Williams, J. M. G., Barnhofer, T., Crane, C., Herman, D., Raes, F., Watkins, E., \& Dalgleish, T. (2007). Autobiographical memory specificity and emotional disorder. Psychological bulletin, 133(1), 122.

Wittchen, H.-U., Wunderlich, U., Gruschwitz, S., \& Zaudig, M. (1997). SKID I. Strukturiertes Klinisches Interview für DSM-IV. Achse I: Psychische Störungen. Interviewheft und Beurteilungsheft. Eine deutschsprachige, erweiterte Bearb. d. amerikanischen Originalversion des SKID I. 
RUNNING HEAD: FUTURE EXPECTATIONS DEPRESSION

\section{Footnotes}

1 Following Dushoof, Kain, Bolker (2018), we use the term statistical clarity instead of statistical significance. The latter may be misleading and prone to misinterpretation. For more details see https://arxiv.org/abs/1810.06387. 